\section{Integrative Medicine \\ International}

\title{
Acupuncture Point Laterality: Evidence and Perspective
}

\author{
Guangjun Wang ${ }^{a}$ Daniela Litscher ${ }^{b-d}$ Yuying Tian ${ }^{a}$ Ingrid Gaischek ${ }^{b-d}$ \\ Shuyong Jia ${ }^{a}$ Lu Wang $^{\text {b-d }}$ Weibo Zhang ${ }^{a}$ Gerhard Litscher ${ }^{\text {a-d }}$ \\ anstitute of Acupuncture and Moxibustion, China Academy of Chinese Medical Sciences, \\ Beijing, China; ${ }^{b}$ Research Unit for Complementary and Integrative Laser Medicine, \\ ${ }^{c}$ Research Unit of Biomedical Engineering in Anesthesia and Intensive Care Medicine, and \\ dTCM Research Center Graz, Medical University of Graz, Graz, Austria
}

\section{Key Words}

Acupuncture point · Acupoint lateralization · Heart rate variability $\cdot$ Blood perfusion

\begin{abstract}
Background: In the Yellow Emperor Neijing, it is stated that 'if someone has a disease related with the left side, the treatment point is on the right side, and vice versa' [Unschuld PU: Huang Di Nei Jing Su Wen, 2003], which emphasizes that specific lateral-side acupoint stimulation might lead to therapeutic advantages under specific conditions. Methods: We summarize the different effects resulting from namesake acupoint stimulation referred to as acupoint laterality. In this short review, the evidence of acupoint lateralization from Neiguan (PC6), Hegu (LI4), and Quchi (LI11) is discussed. Results and Conclusion: Investigations indicate that acupuncture at the contralateral side of PC6 has different effects on heart rate variability (HRV), which means that PC6 has laterality. However, such a difference in HRV could not be observed when the same stimulation was applied to both sides of LI4, although the difference in blood perfusion distribution was confirmed exactly. Considering the results from PC6, we hypothesize that acupoint laterality might be related to acupoint specificity. Although the evidence is not clear, our results indicate that in patients with hypertension, different-side LI11 stimulation can also produce different results, which might indicate that acupoint laterality is associated with the functional state of subjects. The perspective of acupoint lateralization is also preliminarily discussed in this review.

Prof. Gerhard Litscher, MSc, PhD, MDsc TCM Research Center Graz Medical University of Graz AT-8036 Graz (Austria)

E-Mail gerhard.litscher@medunigraz.at
Prof. Weibo Zhang, PhD

Institute of Acupuncture and Moxibustion

China Academy of Chinese Medical Sciences

Beijing 100700 (China)

E-Mail zhangweibo@ hotmail.com 


\section{Introduction}

Acupuncture has been used to treat diseases for at least more than 2,000 years [1]. According to the Huang Di Nei Jing Su Wen [2], acupoint specificity is an essential principle [3, 4], which means that the therapeutic efficacy mainly results from the correct acupoint choice and acupuncture effects are absolutely related to the appropriate acupoint selection during the treatment. Although many researchers believe that a placebo effect is the best explanation for acupuncture [5], acupoint specificity was strengthened by evidence from both clinical practice [6,7] and basic exploration [8].

However, studies usually focus on different acupoints; the difference between namesake acupoints has been ignored in both clinical and experimental settings so far. In the Yellow Emperor Neijing, it is stated that if someone has a disease related with the left side, the treatment point is on the right side, and vice versa', '巨刺' and '缪刺' [2], which emphasizes that specific lateral-side acupoint stimulation might lead to therapeutic advantages under specific conditions. Particularly, this idea has been developed into the concept of contralateral acupuncture in recent years $[9,10]$, and we summarized this as acupoint laterality [11].

The goal of this short review is to discuss the state of the art, the evidence, and the perspective of acupoint laterality.

\section{Evidence of Acupoint Laterality}

Our previous studies have shown that thermostimulation could result in an increase in blood perfusion not only in the local area [12] but also in the same area on the contralateral side [13]. This phenomenon can be observed both in the upper limb [13] and the lower limb [14] but not around the periumbilical area [12]. Research has indicated that bilateral blood perfusion of the same area might be due to special relationships; it has to be taken into account that the distribution of blood perfusion has specificity and the blood flow at the acupoint increases, whereas the blood flow at a nonacupoint only changes slightly by the same stimulation [15]. Our previous study focused on the blood perfusion changes resulting from acupuncture. Another previous study also indicated that acupuncture at the Hegu acupoint (LI4) significantly increased perfusion at this acupoint but not at a nearby nonacupoint [16]. Thus, firstly, we observed a blood perfusion change in LI4 after the contralateral LI4 was stimulated. Totally, 140 healthy volunteers were recruited and the results indicated that either unilateral LI4, stimulated by manual acupuncture, caused the blood perfusion in the contralateral LI4 to increase significantly during $150 \mathrm{~min}$ after stimulation [17]. This work was partly supported by Kubo et al. [18]. However, stimulation of the LI4 acupoint with a metal needle will produce insufferable pain and is accompanied by whole-body reactions. Recently, the laser needle as an alternative method to manual acupuncture has been used in many studies because it is effective not only as a treatment method but also as a research tool [19-21]. It can simulate traditional acupoints while also reducing the stress effect resulting from metal needles. In order to eliminate the noise effect resulting from manual acupuncture, needle acupuncture was replaced by laser acupuncture. The blood perfusion of the left LI4 increased after the right LI4 was stimulated. Wavelet analysis results suggested that the increased blood perfusion might be related to the endothelial, neurogenic, and intrinsic myogenic activity of the vessel wall [22].

Unilateral LI4 stimulation on either side resulted in blood perfusion changes in the contralateral LI4; but what are the differences between the sides? In other words, are the effects the same, or how can they be differentiated? To answer this question, a system identification algorithm was applied to analyze blood perfusion, and the results suggested that differences exist between the left and right LI4. The function of the namesake LI4 is asymmetric [23]. 
Fig. 1. Either-side acupoint manual stimulation might result in different changes in HRV in healthy subjects and hypertensive patients.

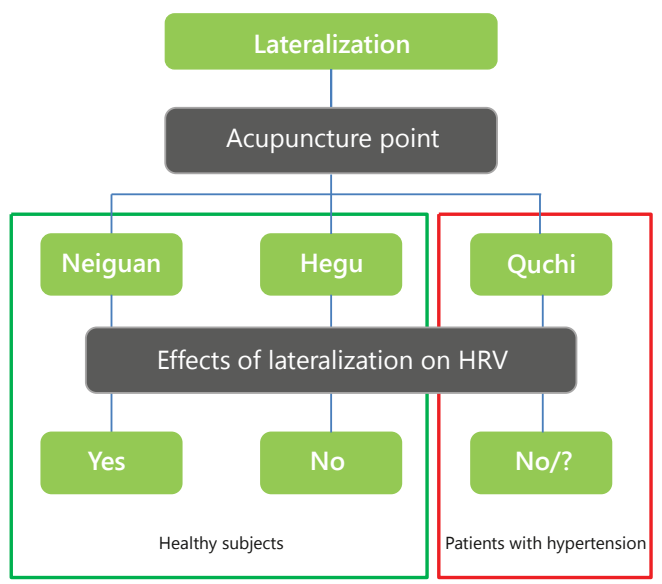

According to traditional acupuncture theory, the function of acupuncture points is limited. Given that an acupoint can treat all disorders, the basis for acupoint specificity might disappear. LI4 belongs to the large intestine meridian, which means LI4 has little or no effect on cardiac symptoms. On the other hand, Neiguan (PC6) belongs to the pericardium meridian and is usually used as a decisive acupoint in cardiac symptom treatment. Nevertheless, unilateral effects of both PC6 and LI4 on heart rate variability (HRV) have been observed. In these studies, healthy subjects were recruited and a cross-over design was applied. Consistent with our expectations, the different sides of PC6 have different effects on HRV [24], while LI4 does not have these effects [25]. Recently, the laterality of Quchi (LI11) has also been explored (fig. 1) [26]. Although the evidence is not clear, our results indicate that in patients with hypertension, different-side LI11 stimulation can also produce different results, which might indicate that acupoint laterality is associated with the functional state of subjects [26].

\section{Perspective of Acupoint Laterality}

At the behavioral level, lateralization is expressed in side biases for perception, motor output, and information processing. For a long time, lateralization was considered unique to humans, but recently, it has become clear that lateralization is a fundamental characteristic of the organization of the brain and behavior in vertebrates [27] and invertebrates [28]. Not only is lateralization common to vertebrates, but there also appears to be a common pattern of lateralization, which has been retained amongst all of the major groups of vertebrates [29-31]. Lateralization of the brain and behavior usually refers to the fact that the hemispheres of the brain control behavior differentially. It is also known as hemispheric or cerebral specialization [27]. For acupoint lateralization, we refer to the functional specialization of namesake acupoints under certain conditions. Some clues of acupuncture point lateralization have been captured by our very preliminary studies, and the important role of these clues in promoting the development of acupuncture has been appreciated by previous studies. However, the issue of acupoint lateralization has not yet been entirely elucidated. We believe that answering only the following questions might shed light on the uncertainties surrounding acupoint lateralization.

The first question is the relationship between acupoint lateralization and handedness. We do not know whether acupoint lateralization is an independent characteristic or a char- 
acteristic associated with handedness. According to a survey, only $7.6 \%$ of the children of two right-handed parents are left-handed. The percentage of left-handed children increases to $19.5 \%$ if one of the parents is left-handed and to $54.5 \%$ if both parents are left-handed [32]. The survey results suggest that handedness is closely related to the genetic background. On the other hand, although offspring of left-handed parents are more likely to be left-handed than offspring of right-handed parents, right-handed offspring can be produced by two lefthanded parents, which means that the genetic background is not the only decisive factor in the epigenesis of handedness. Similarly, the epigenetic factor of acupoint lateralization should be explored. By describing correlations between genetic information, environmental factors, and the development or expression of lateralization, or by manipulating genetic and environmental factors using animal models, such insights can be gained.

The second question is whether acupoint lateralization is a phenomenon at the population level or at the individual level. So far, the survey data on acupoint lateralization are still scarce. According to some views, if the population is not lateralized even though individuals are, the evolutionary significance is debatable, and, therefore, some scientists prefer to concentrate only on lateralization at the population level. However, although acupoint lateralization is reflected in the individual, we believe that the potential therapeutic value of lateralization should be further explored.

The third question is whether this lateralization can be changed under special conditions. In biology, fluctuating asymmetries, consisting of random deviations from bilateral symmetry in individuals, have been described for a number of different species, including fish. They are associated with environmental stress or reduced heterozygosis and are believed to be due to the incapacity of individuals to undergo identical development on both sides of the body [33]. From our results, LI4 has no lateralization effects on HRV in healthy subjects, whereas LI11 has lateralization effects on HRV in patients with hypertension. We think lateralization might also fluctuate under certain conditions.

In our opinion, these three questions are directly related to the core of acupoint lateralization. Only if these questions will be answered, will acupoint lateralization truly be considered; in standardized clinical practice, acupuncture point selection may become more accurate and optimized, and the point prescription may be more reasonable.

\section{Acknowledgements}

This research was supported by the National Natural Science Foundation of China (81001553) and the Fundamental Research Funds for the Central Public Welfare Research Institutes (ZZ070806). The scientific investigations were also supported by the Austrian Federal Ministries of Science, Research and Economy and of Health (project title 'Evidencebased high-tech acupuncture and integrative laser medicine for prevention and early intervention of chronic diseases') and the German Academy of Acupuncture (DAA). Assoc. Prof. Guangjun Wang is currently working at the Medical University of Graz with a scholarship from Eurasia Pacific Uninet. The authors would also like to thank Prof. Weiping Cheng from the First Hospital affiliated to the Heilongjiang University of Chinese Medicine, Harbin, China, for performing a part of the investigations in patients at his department.

\section{Disclosure Statement}

The authors declare that there are no conflicts of interest concerning the publication of this article. 


\section{References}

1 Lu GD, Needham J: Celestial Lancets: A History and Rationale of Acupuncture and Moxa. Cambridge, Cambridge University Press, 1980.

2 Unschuld PU: Huang Di Nei Jing Su Wen: Nature, Knowledge, Imagery in an Ancient Chinese Medical Text, with an Appendix: The Doctrine of the Five Periods and Six Qi in the Huang Di Nei Jing Su Wen. Berkeley, University of California Press, 2003.

3 Zhao L, Chen J, Liu CZ, Li Y, Cai DJ, Tang Y, Yang J, Liang FR: A review of acupoint specificity research in China: status quo and prospects. Evid Based Complement Alternat Med 2012;2012:543943.

4 Rong PJ, Zhao JJ, Gao JH, Li X, Li SY, Ben H, Pearson B: Progress of research on specificity of meridian acupoint efficacy. Chin J Integr Med 2013;19:889-893.

5 Wechsler ME, Kelley JM, Boyd IOE, Dutile S, Marigowda G, Kirsch I, Israel E, Kaptchuk TJ: Active albuterol or placebo, sham acupuncture, or no intervention in asthma. N Engl J Med 2011;365:119-126.

-6 Yang J, Zeng F, Feng Y, Fang L, Qin W, Liu X, Song W, Xie H, Chen J, Liang F: A PET-CT study on the specificity of acupoints through acupuncture treatment in migraine patients. BMC Complement Altern Med 2012;12: 123.

7 Zhao L, Liu J, Zhang F, Dong X, Peng Y, Qin W, Wu F, Li Y, Yuan K, von Deneen KM, et al: Effects of long-term acupuncture treatment on resting-state brain activity in migraine patients: a randomized controlled trial on active acupoints and inactive acupoints. PLoS One 2014;9:e99538.

-8 Ren YS, Bai LJ, Feng YY, Tian J, Li KC: Investigation of acupoint specificity by functional connectivity analysis based on graph theory. Neurosci Lett 2010;482:95-100.

-9 Kim MK, Choi TY, Lee MS, Lee H, Han CH: Contralateral acupuncture versus ipsilateral acupuncture in the rehabilitation of post-stroke hemiplegic patients: a systematic review. BMC Complement Altern Med 2010; 10:41.

10 Woo YM, Lee MS, Nam Y, Cho HJ, Shin BC: Effects of contralateral electroacupuncture on brain function: a double-blind, randomized, pilot clinical trial. J Altern Complement Med 2006;12:813-815.

$\checkmark 11$ Wang GJ: Study on acupoint laterality: the important supplement to acupoint specificity. Zhongguo Zhen Jiu 2012;32:709-712.

12 Wang GJ, Zhang YQ, Wang RH, Ding YL, Tian YY, Huang T, Zhang WB: Experimental study on effect of electro-heated stone needle on local skin blood perfusion of dorsum hand. Tianjin Zhong Yi Yao 2009;26: 382-384.

13 Wang GJ, Zhang YQ, Wang RH, Ding YL, Tian YY, Huang T, Zhang WB: The study of interaction based on the thermostimulation. Chin J Basic Med Trad Chin Med 2010;16:803-804, 811.

14 Zhang YQ, Ding YL, Tian YY, Huang T, Zhang WB, Wang GJ: Change of blood perfusion on contra-lateral lower limb after electro-bian stone intervention. Jiangsu Zhong Yi Yao 2010;42:48-49.

15 Kuo TC, Chen ZS, Chen CH, Ho FM, Lin CW, Chen YJ: The physiological effect of De Qi during acupuncture. J Health Sci 2004;50:336-342.

16 Hsiu H, Hsu WC, Hsu CL, Huang SM: Assessing the effects of acupuncture by comparing needling the Hegu acupoint and needling nearby nonacupoints by spectral analysis of microcirculatory laser Doppler signals. Evid Based Complement Alternat Med 2011;2011:435928.

17 Wang GJ, Tian Y, Jia S, Huang T, Zhang W: Change of blood perfusion in Hegu acupoint after contralateral Hegu acupoint was stimulated. J Altern Complement Med 2012;18:784-788.

18 Kubo K, Yajima H, Takayama M, Ikebukuro T, Mizoguchi H, Takakura N: Changes in blood circulation of the contralateral Achilles tendon during and after acupuncture and heating. Int J Sports Med 2011;32:807813.

19 Litscher G, Opitz G: Technical parameters for laser acupuncture to elicit peripheral and central effects: state-of-the-art and short guidelines based on results from the Medical University of Graz, the German Academy of Acupuncture, and the scientific literature. Evid Based Complement Alternat Med 2012;2012: 697096.

20 Litscher G, Bauernfeind G, Mueller-Putz G, Neuper C: Laser-induced evoked potentials in the brain after nonperceptible optical stimulation at the Neiguan acupoint: a preliminary report. Evid Based Complement Alternat Med 2012;2012:292475.

-21 Litscher G: Integrative laser medicine and high-tech acupuncture at the Medical University of Graz, Austria, Europe. Evid Based Complement Alternat Med 2012;2012:103109.

-22 Wang G, Tian Y, Jia S, Litscher G, Zhang W: Evaluate laser needle effect on blood perfusion signals of contralateral Hegu acupoint with wavelet analysis. Evid Based Complement Alternat Med 2012;2012:103729.

23 Wang G, Han J, Litscher G, Zhang W: System identification algorithm analysis of acupuncture effect on mean blood flux of contralateral Hegu acupoint. Evid Based Complement Alternat Med 2012;2012:951928.

24 Wang G, Tian Y, Jia S, Zhou W, Zhang W: Pilot study of acupuncture point laterality: evidence from heart rate variability. Evid Based Complement Alternat Med 2013;2013:476064.

25 Wang GJ, Tian Y, Jia S, Zhou W, Zhang W: Bilateral Hegu acupoints have the same effect on the heart rate variability of the healthy subjects. Evid Based Complement Alternat Med 2014;2014:106940.

-26 Litscher G, Cheng WP, Cheng GY, Wang L, Zhao J, Litscher D, Gaischek I, Sheng Z, Kuang H: Acupuncture point laterality: investigation of acute effects of Quchi (LI11) in patients with hypertension using heart rate variability. Evid Based Complement Alternat Med 2014;2014:979067. 
27 Vallortigara G, Rogers LJ: Survival with an asymmetrical brain: advantages and disadvantages of cerebral lateralization. Behav Brain Sci 2005;28:575-589, discussion 589-633.

28 Frasnelli E: Brain and behavioral lateralization in invertebrates. Front Psychol 2013;4:939.

29 Vallortigara G, Rogers LJ, Bisazza A: Possible evolutionary origins of cognitive brain lateralization. Brain Res Rev 1999;30:164-175.

-30 Fitch WT, Braccini SN: Primate laterality and the biology and evolution of human handedness: a review and synthesis. Ann NY Acad Sci 2013;1288:70-85.

-31 Oleksiak A, Postma A, van der Ham IJ, Klink PC, van Wezel RJ: A review of lateralization of spatial functioning in nonhuman primates. Brain Res Rev 2011;67:56-72.

32 Rife DC: Handedness, with special reference to twins. Genetics 1940;25:178-186.

-33 Leary RF, Allendorf FW: Fluctuating asymmetry as an indicator of stress: implications for conservation biology. Trends Ecol Evol $1989 ; 4: 214-217$. 